Écrire

l'histoire

\section{Écrire l'histoire}

Histoire, Littérature, Esthétique

\section{9 | 2019}

\section{L'historien et les langues}

\title{
Toutes terres sont païs au proudome. Les langues de Brunetto Latini
}

Florent Coste

\section{(2) OpenEdition \\ Journals}

Édition électronique

URL : http://journals.openedition.org/elh/1932

DOI : $10.4000 /$ elh. 1932

ISSN : 2492-7457

Éditeur

CNRS Éditions

\section{Édition imprimée}

Date de publication : 1 décembre 2019

Pagination : 109-116

ISBN : 978-2-271-12967-3

ISSN : $1967-7499$

\section{Référence électronique}

Florent Coste, «Toutes terres sont païs au proudome. Les langues de Brunetto Latini », Écrire l'histoire [En ligne], 19 | 2019, mis en ligne le 01 décembre 2019, consulté le 16 février 2021. URL : http:// journals.openedition.org/elh/1932 ; DOI : https://doi.org/10.4000/elh.1932 


\section{Toutes terres sont païs au proudome. Les langues de Brunetto Latini}

Les historiens travaillent dans et sur des langues différentes. Il arrive cependant que les langues de l'historien ne se confondent pas avec celles des acteurs sur lesquels il enquête ou celles des sources qui les documentent. À cela s'ajoutent des traditions historiographiques ou des champs disciplinaires tendant de manière anachronique ou chauvine à projeter sur leurs objets des rapports de force linguistiques qui ne leur sont pas congruents. Cela est saisissant quand les acteurs, particulièrement polyvalents, polyglottes et plurilingues, circulent entre des domaines linguistiques où les historiens naviguent moins aisément et tendent au contraire volontiers à les enfermer. En réalité, les acteurs ne se répartissent pas par avance dans les cases disciplinaires ou linguistiques qui seront celles des historiens qui les étudient.

Ces enjeux s'avivent encore un peu plus à mesure que la période étudiée est plus lointaine. Ainsi du Moyen Âge : car la maîtrise de langues anciennes et moins immédiatement intelligibles comporte un coût plus important pour celui qui l'étudie; mais encore parce qu'il est facile de parcelliser l'histoire médiévale des langues en autant de petites provinces vernaculaires surmontées par une grande langue véhiculaire et sacrée que les acteurs doivent employer s'ils souhaitent s'affranchir de leur langue locale. L'univers des langues au Moyen Âge est beaucoup plus fluide et interactif $\mathrm{qu}^{\prime}$ on voudrait bien l'imaginer : la diglossie y est tempérée par des formes étonnantes de plurilinguisme et des chaînes de traduction plus complexes ${ }^{1}$.

On voudrait succinctement s'emparer ici du cas symptomatique d'un acteur dont le plurilinguisme a fait la centralité en son temps autant que la difficulté aujourd'hui à ne pas le confiner dans des angles morts historiographiques. Il s'agit de Brunetto Latini, un laïc florentin du XIII $^{\mathrm{e}}$ siècle dont la carrière tumultueuse et la polyvalence intellectuelle ont démultiplié et dispersé ses traces en latin, en langue d'oïl et en volgare. 


\section{La carrière d'un intellectuel laïc au carrefour des langues}

Né dans les années 1220 et mort en 1294, Brunetto Latini est un de ces personnages aux coordonnées multiples qui débordent et transcendent largement nos découpages génériques, linguistiques et disciplinaires. Le chroniqueur florentin Giovanni Villani vantait en lui un homme de culture qui avait assuré au bénéfice de toute sa cité une éducation civique de la parole : il fut un "gran filosofo ", " cominciatore e maestro in digrossare $i$ Fiorentini e farli scorti in bene parlare, e in sapere guidare e reggere la nostra repubblica secondo la politica». À ce «sommo maestro in rettorica, tanto in bene sapere dire come in bene dittare ${ }^{2}$ ( (entendons l'art oratoire et l'art épistolaire du dictamen), on doit en effet une œuvre indissociablement rhétorique, savante et politique: le Tresor composé en langue d'oïl s'est imposé comme une compilation encyclopédique faisant office de guide du bon gouvernement au service de la refondation d'une citoyenneté postféodale et communale ; dédié à Charles d'Anjou, le Tesoretto en est une version versifiée, volgarizzata, réaménagée dans l'horizon ouvert par la victoire guelfe après la bataille de Benevento ${ }^{3}$; ce poème didacticoallégorique est par ailleurs parfois suivi dans ses manuscrits par le Favolello, une pièce poétique sur l'amitié ${ }^{4}$; il réalise également des volgarizzamenti des trois discours (Pro Ligario, Pro Marcello, Pro rege Deiotaro) que Cicéron adresse à César après sa victoire dans la guerre civile $^{5}$; la Rettorica propose enfin une traduction actualisante du De inventione de Cicéron. Le titre de cette œuvre est à lui seul symptomatique des pouvoirs éminemment politiques que Brunetto prête à la parole : dans la graphie rettorica se confondent le rhéteur (chargé de l'art oratoire) autant que le recteur (chargé de l'art de gouverner).

Au-delà de l'œuvre qu'il a pu constituer, les sources laissent apparaître d'autres traces de sa présence et des offices qu'il a accumulés : attesté comme notarius, judex, cancellarius, parfois désigné comme sindaco de la commune de Florence, il est aussi protonotaire du vicaire de Charles d'Anjou en Toscane et à plusieurs reprises ambassadeur de Florence $^{6}$. Il importe ainsi de compter sur son investissement en latin dans l'écriture de chancellerie, à savoir dans le genre indissociablement littéraire et politique du dictamen, où il endosse, non sans admiration, l'héritage du stilus obscurus des Lettres de Pierre de la Vigne ${ }^{7}$. Aussi se cristallise en lui la figure complète d'un laïc litteratus, posté à l'intersection du latin, du français et de l'italien, en qui se concilient le citoyen polyvalent et polyglotte impliqué dans la refonte de nouveaux savoirs tout autant que le notaire engagé dans la gestion courante des affaires politiques ${ }^{8}$.

Les profils historiographiques de Brunetto Latini n'en sont que plus variés, et difficiles à recomposer. Tantôt il est, pour les philosophes et les historiens de la pensée politique, l'initiateur d'une laïcisation de la philosophie émancipée de la langue latine des théologiens, ou encore la figure d'un républicanisme qui a œuvré à la réinvention d'un nouveau Cicéron pour poser les jalons d'une idéologie du bien 
commun avant la redécouverte d'un Aristote politique ${ }^{9}$. Tantôt il est, pour les historiens de la littérature et de la langue italiennes, l'un des initiateurs d'une politique du volgarizzamento, parce qu'il est en l'occurrence le premier à traduire Cicéron et à commenter un texte latin en langue vulgaire ${ }^{10}$. À l'échelle de l'histoire de Florence, cette fois, il fait bonne figure face à Dante, Coluccio Salutati ou Niccolò Machiavelli, dont certains se figurent qu'il est comme une anticipation. Enfin, dans la perspective de l'histoire de la langue française, Brunetto est "posto coscientemente sull'onda dell'espansione linguistica francese ${ }^{11} »:$ avec $\mathrm{d}^{\prime}$ autres
Italiens francophones du Moyen Âge (comme Martino da Canale, Niccolò da Verona, Rustichello da Pisa et Marco Polo, Daniele Deloc, Filippo di Novara), il offre un témoignage probant et largement remobilisé de la valeur culturelle dont se pare le français véhiculaire au-delà du royaume de France dans la Méditerranée médiévale ${ }^{12}$.

Comme on s'en doute, ces champs historiographiques, loin d'être compartimentés, doivent s'articuler, et nécessitent notamment de frayer une voie moyenne entre une historiographie qui francise trop Brunetto Latini et une autre qui l'italianise au contraire avec les mêmes excès.

\section{Brunetto Latini à l'ombre et à la lumière de Dante}

Brunetto précède Dante d'une génération. Il est d'ailleurs le maître et l'initiateur de l'Alighieri, dont l'ombre portée conditionne fatalement le regard que nous portons sur le notaire. Le poète florentin a contribué à faire connaître la figure de Brunetto Latini en l'entourant un peu plus d'une aura énigmatique. Dante le retrouve dans le troisième giron du septième cercle de l'Enfer, où il l'a placé à essuyer une pluie de flammes avec ceux qui ont fait preuve de violence contre la Nature, à savoir les sodomites ${ }^{13}$. Dante garde pourtant un attachement ému pour cette figure paternelle (la cara e buona imagine paterna), qui lui apparaît la figure cuite (lo cotto aspetto) et le visage brûlé ('l viso abbrusciato). La condamnation de Brunetto et son assignation au cercle des sodomites ont suscité de nombreuses interrogations parmi les spécialistes de Dante. Parmi eux, André Pézard a jadis émis l'hypothèse, encore discutée, selon laquelle la faute contre nature de Brunetto aurait consisté à avoir utilisé et magnifié une langue autre que sa langue maternelle. La condamnation devrait ainsi être mise sur le compte d'un dissensus non pas tant politique (il est vrai, l'un est guelfe, l'autre gibelin) que linguistique et poétique entre un élève qui avait soutenu la supériorité des langues vulgaires $^{14}$ et le maître ne semblant pas se satisfaire de la langue italienne, à laquelle, quand il l'employait, il ajoutait des occitanismes ${ }^{15}$.

Sans entrer dans de trop vastes débats, on peut affirmer que le couple intellectuel que forment Brunetto Latini et Dante est à bien des égards exemplaire des rapports de force en pleine mutation qui se laissent entrevoir à la charnière du Duecento et du Trecento entre la langue d'oil et le volgare. Dans le prologue du 
Tresor, Brunetto se justifie de son usage du français: au-delà de sa situation d'exilé en France, il invoque les qualités esthétiques que l'on prête au français et son statut de langue véhiculaire :

Et se aucun demandoit por quoi ceste livre est escrit en roman selonc le patois de France, puis que nos sommes ytaliens, je diroie que ce est par.ii. raisons : l'une que nos sommes en France, l'autre por ce que la parleure est plus delitable et plus comune a touz languaiges ${ }^{16}$.

Langue commune, le français représente pour Brunetto Latini une garantie d'accès à des publics non locaux et un gisement de sources de grande valeur dans lesquelles puiser et compiler ${ }^{17}$. Il $\mathrm{s}^{\prime}$ agit bien de cette langue que convoque également Martino da Canale dans ses Estoires de Venise pour la raison qu'elle "cort parmi le monde", tant sur les routes de commerce et de pèlerinage qu'avec les croisés qui s'installent dans les États latins d'Orient, et qu'elle permet de capter des publics autrement inaccessibles. Il faut d'ailleurs remarquer à cet égard que l'Italie a joué un rôle essentiel de plate-forme et de relais dans la constitution et la propagation de traditions manuscrites méditerranéennes dans un français dit d'Outremer, dont le Tresor a pu bénéficier ${ }^{18}$. Brunetto exploite donc les réseaux internationaux et méditerranéens d'une langue véhiculaire à succès pour assurer une efficace diffusion de son œuvre ${ }^{19}$; mais la réciproque est également vraie: tout autant, le Livre dou Tresor semble s'être imposé comme l'une des courroies de transmission de cette expansion hors de France de la langue d'oïl. Dans ce plaidoyer pour le primat des parlers vulgaires et pour la conquête d'un italien aulique qu'est le De vulgari eloquentia, Dante explique les raisons qui président au succès de la langue française, en illustrant les œuvres qui incarnent ce prestige :

Allegat ergo pro se lingua oül, quod propter suifaciliorem ac delectabiliorem vulgaritatem quicquid redactum sive inventum est ad vulgare prosaycum, suum est : videlicet Biblia cum Troianorum Romanorumque gestibus compilata et Arturi regis ambages pulcerrime et quamplures alie ystorie ac doctrine $^{20}$.

Langue de culture plaisante et agréable, le parler d'oïl excelle entre toutes dans la prose, à savoir dans les romans arthuriens où la militia des communes et des seigneuries italiennes se forge sa culture chevaleresque, dans les compilations historiographiques comme l'Histoire ancienne jusqu'à César, le Roman de Troie et les Faits des Romains, enfin dans «bien d'autres ouvrages d'histoire et de science » - et il est probable que Dante pense là précisément au Tresor compilé par son maître.

L'écart générationnel entre Brunetto et Dante est celui-là même qui sépare une situation sociolinguistique où l'italien volgare encore dominé par un français international est tout juste in statu nascendi $i^{21}$ et un contexte où l'italien s'est approprié grâce à Dante de grandes capacités d'expression poétique et philosophique. Reste quel'inversion de ce rapport de force doit beaucoup à Brunetto, dont la Rettorica et les volgarizzamenti du Tresor ont impulsé en Toscane un mouvement d'appropriation du parler vulgaire italien ${ }^{22}$. 


\section{Diplomatie, exil et plurilinguisme}

La capacité de Brunetto à écrire dans trois langues, à travailler dans une polyglossie par lui assouplie, aussi bien qu'à faire œuvre de translation, l'a mis en situation d'articuler et de recomposer des mondes hétérogènes. On sait qu'il fut aussi un émissaire envoyé en ambassade par la commune de Florence, d'abord pour convaincre Alphonse $X$ de Castille de revendiquer la couronne impériale et de contrer ainsi Manfred et les gibelins, plus tard auprès de Gênes en $1284^{23}$. C'est sur le chemin du retour de son ambassade en Espagne qu'il apprend la défaite de la Florence guelfe contre la Sienne gibeline à la bataille de Montaperti en 1260 : il fait le récit dans le Tesoretto de la rencontre d'un étudiant bolognais qui, sur la plaine de Roncevaux - lieu par excellence de la défaite -, lui apprend la nouvelle dans sa douce langue de Toscane (in dolce lingua e piana ${ }^{24}$ ). Son retour en Toscane ainsi empêché provoque son exil en France entre 1260 et 1265. De cette période difficile d'émigration, nous gardons la trace par ses activités de notaire pour des compagnies de marchands florentins à Bar-sur-Aube, à Arras ou à Paris (où se pose la question de savoir s'il mit un pied à la Sorbonne). Que cette situation d'exil politique fut féconde et n'en aviva que davantage ses options linguistiques, on ne peut guère en douter. La première raison de composer le Tresor en français est, dit-il, que «nous sommes en France ». Au cœur de son livre II, Brunetto orchestre d'ailleurs un dialogue entre Paor et Seurtez, et, à la menace que la première brandit de chasser la seconde en exil, cette dernière répond :

Toutes terres sont païs au proudome, autresi come la mer au poisson. Ou que je aille, serai je a la moie terre, que terre nulle ne est exil, mes estrange païs ${ }^{25}$.

Le proudome - l'homme de valeur ne connaît pas de terre absolument étrangère dont la langue lui serait parfaitement inintelligible. De ce point de vue, l'ambassade de Brunetto à l'été 1260 auprès d'Alphonse le Sage fait l'objet de nombreuses conjectures quant aux œuvres que le notaire florentin a pu voir et ramener de Tolède, animée par une école de traduction très active dans la dissémination de textes arabes : rapporta-t-il et importa-t-il la Politique $\mathrm{d}$ 'Aristote en Occident? Croisa-t-il les traducteurs du mi rāj, le récit de l'ascension du Prophète au paradis, amené à devenir en latin le Livore de l'Échelle de Mahomet? S'il faut s'en tenir prudemment au stade de la conjecture et ne pas céder au fantasme du passeur héroïque entre les mondes ${ }^{26}$, il est bon de souligner que ce récit de l'eschatologie musulmane fut d'abord traduit de l'arabe au castillan par un médecin juif prénommé Abraham, puis du castillan au latin par un Toscan, Bonaventura da Siena, par ailleurs notaire et secrétaire du roi, qu'il serait étonnant que Brunetto n'ait pas croisé durant son séjour à Tolède ${ }^{27}$. 
La difficulté est grande de ne pas mutiler les multiples visages de Brunetto, tout comme de ne pas l'inféoder de manière téléologique à une histoire de la langue italienne dont Dante serait le monumental seuil d'entrée, ou encore aux premiers moments d'un inéluctable humanisme. Ce notaire polygraphe qu'est Brunetto nous rappelle qu'il se situe au moment charnière mais incertain où la question des langues, dans leurs

\section{Notes}

1 Benoît Grévin, Le Parchemin des cieux. Essai sur le Moyen Âge du langage, Seuil (L'univers historique), 2012.

2 Cronica di Giovanni Villani a miglior lezione ridotta, Florence, 1823, VIII, 10, vol. 3, p. 22.

3 Gianluca Briguglia (" "Io, Burnetto Latini". Considerazioni su cultura e identità politica di Brunetto Latini e il Tesoretto ", Philosophical Readings, vol. X, no 3, 2018, p. 176-185, DOI : 10.5281/zenodo.1467865) présente le Tesoretto comme un "tentativo di costruire una cultura politica rinnovata, attraverso un progetto pedagogico di articolazione di valori compatibili fra le varie componenti della nuova Firenze » (p. 183).

4 Voir le commentaire et l'édition de Patrizia GASPARINI, «L'amitié comme fondement de la concordia civium », Arzanà, n 13,2010 , p. 55-108, DOI : 10.3406/arzan.2010.970.

5 Cicéron, Pro Ligario, Pro Marcello, Pro rege Deiotaro (Orazioni cesariane). Volgarizzamento di Brunetto Latini, édit. Cristiano Lorenzi, Pise, Edizioni della Normale, 2018.

6 Roberta Cella, «Gli atti rogati da Brunetto Latini in Francia (tra politica e mercatura, con qualche implicazione letteraria)", Nuova Rivista di letteratura italiana, vol. 6, n ${ }^{\circ} 1-2,2003$, p. 367-408, DOI : 10.4454/nrli.v6i1-2.125; Giorgio INGLESE, "Latini, Brunetto », dans Dizionario biografico degli Italiani, vol. 64, 2005.

7 Benoît Grévin, Rhétorique du pouvoir médiéval. Les Lettres de Pierre de la Vigne et la formation du contacts et dans leurs rapports de force dynamiques, recoupe étroitement celle de l'invention d'une nouvelle culture politique et s'inscrit dans cette conjoncture où l'Italie communale se cherche, somnambule et tâtonnante, un langage politique ${ }^{28}$. L'histoire des langues ne doit pas, on le comprend, plaquer sa logique souvent internaliste et volontiers involutive sur l'histoire tumultueuse de leurs locuteurs. langage politique européen (XIII ${ }^{e}-\mathrm{XV}{ }^{e}$ siècle), École française de Rome, 2008, DOI : 10.4000/books. efr.479, p. 823-825. Brunetto témoigne de son admiration pour Pierre de la Vigne dans sa Rettorica, au moment de son exil, quand il n'a plus les modèles latins sous la main: Pierre de la Vigne y est présenté comme un éminent "dittatore, il quale perciò fue agozetto di Federigo secondo imperadore di Roma e tutto sire di lui e dello'impero ». La rhétorique relève selon lui de l'art oratoire (discours oral) et de l'art épistolaire (discours écrit). "Rettorica è scienza di due manière: una la quala insegna dire, e di questa tratta Tulio nel suo libro; l'altra insegna dittare, $e$ di questa, perciò che esse non ne trattò così del tutto apertamente, si nne tratterà la sponitore nel processo del libro, in suo luogo e tempo come si converrà » (BRUNeTto LATINI, La Rettorica, édit. Maggini, 1968, p. 1 et 3).

8 Emanuele Coccia, Sylvain Piron, «Poésie, sciences et politique. Une génération d'intellectuels italiens (1290-1330) ", Revue de synthèse, vol. $129,6^{\text {e }}$ série, $\mathrm{n}^{\circ} 4,2008$, p. 549-586, DOI : 10.1007/s11873-008-0061-4.

9 Quentin SKInNER, Les Fondements de la pensée politique moderne [1979], traduit de l'anglais par Jerome Grossman et Jean-Yves Pouilloux, A. Michel, 2001. Pour une réarticulation entre histoire politique et histoire de la pensée politique, voir les travaux d'Enrico ArTIFOnI, «Una politica del dittare: l'epistolografia nella Rettorica di Brunetto Latini », dans Paolo Cammarosano et al. (dir.), Art de la lettre et Lettre d'art. Épistolaire politique III, Trieste, CERM/Rome, École française de Rome, 2016, 
p. 175-194. Pour une réévaluation de la figure de Cicéron dans l'histoire communale italienne, voir Carole Mabboux, Cicéron et la Commune. Présence(s) d'une autorité rhétorique et politique dans la culture civique italienne (XIII-XIV siècles), École française de Rome, BEFAR, à paraître.

10 Voir par exemple Alison CoRNISH, Vernacular Translation in Dante's Italy. Illiterate Literature, Cambridge University Press, 2011.

11 Cesare Segre, Mario Marti (dir.), La prosa del Duecento, Milan/Naples, R. Ricciardi, 1959, p. 311.

12 Il s'agit là d'un champ récent et particulièrement dynamique dans la médiévistique européenne. Voir entre autres Christopher KLeInHenz, Keith Busby (dir.), Medieval Multilingualism. The Francophone World and Its Neighbours, Turnhout, Brepols, 2010 ; et The Values of French Language and Literature in the European Middle Ages, ERC Advanced Grant dirigé par Simon Gaunt (King's College London), <https:// tvof.ac.uk/>. Sur le franco-italien au Moyen Âge, voir toutes les contributions du premier fascicule 2015 de Medioevo Romanzo. Au début $\mathrm{du} \mathrm{xx}^{\mathrm{e}}$ siècle, cette question intéressait déjà les philologues, selon une conception encore concentrique et expansionniste (Paul Meyer, De l'expansion de la langue française en Italie pendant le Moyen Âge, Rome, Reale Accademia dei lincei, 1904). Il est bon de rappeler enfin que dès la fin du XVIII $^{\mathrm{e}}$ siècle Rivarol brandissait pour preuve du génie universel de la langue française la figure de Brunetto Latini - «un auteur italien [qui a] trouvé assez de charmes [à la langue française] pour la préférer à la sienne » (Antoine de Rivarol, De l'universalité de la langue française, Paris, Bailly et Dessenne, 1784 , p. 4).

13 Inferno, 15, 22-24, v. 79-87.

14 Sur le primat du vulgaire chez Dante, voir De l'éloquence en vulgaire, introduction et appareil critique par Irène Rosier-Catach, traduction française par Anne Grondeux, Ruedi Imbach et Irène Rosier-Catach, Fayard, 2011, I, I, 4 : « nobilior est vulgaris: tum quia prima fuit humano generis usitata; tum quia totus orbis ipsa perfruitur ». Sur la critique par Dante des Italiens qui, en s'adonnant à d'autres langues, déprécient l'italien, voir Il Convivio, I, XI : «A perpetuale infamia e depressione de li malvagi uomini d'Italia commendano lo volgare altrui e lo loro proprio dispregiano. »
15 André PÉzArd, Dante sous la pluie de feu. Enfer, chant XV, J. Vrin, 1950. Pour d'autres interprétations, voir Serge VANVOLSEM, «Brunetto Latini, lingua di cultura e lingua dell'emigrazione ", dans François Livi (dir.), De Marco Polo à Savinio. Écrivains italiens en langue française, Presses de l'université Paris-Sorbonne, 2003, p. 21-33 ; Alison Cornish, op. cit., chap. 5.

16 Brunetto Latini, Tresor, édit. P. G. Beltrami, P. Squillacioti, P. Torri et S. Vatteroni, Turin, G. Einaudi, 2007, I, 1, 7, p. 6.

17 Brunetto puise dans l'historiographie en langue d'oïl, d'une part dans l'Histoire ancienne jusqu'à César (Maria Teresa Rachetta, "Sulla sezione storica del Tresor: Brunetto Latini e l'Histoire ancienne jusqu'à César", Medioevo Romanzo, 2018, p. 284-311), d'autre part dans les Faits des Romains, une compilation et une traduction en langue d'oïl des principales sources latines sur la vie de César (Salluste, Lucain, César lui-même) qu'il utilise pour construire avec Cicéron une figure de l'orateur modèle (Ronald G. WitT, L'eccezione italiana. L'intellettuale laico nel Medioevo e l'origine del Rinascimento (800-1300), Rome, Viella, 2017, p. 524-527; traduction de The Two Latin Cultures and the Foundation of Renaissance Humanism in Medieval Italy, Cambridge/New York, Cambridge University Press, 2012).

18 Fabio Zinelli, "Sur les traces de l'atelier des chansonniers occitans IK: le manuscrit de Vérone, Biblioteca Capitolare, DVIII et la tradition méditerranéenne du Livre dou Tresor", Medioevo Romanzo, 2007, p. 7-69; ID., "Tradizione "mediterranea" e tradizione italiana del Livre dou Tresor », dans Irene MAfFIA ScARIATI (dir.), A scuola con Ser Brunetto. Indagini sulla ricezione di Brunetto Latini dal Medioevo al Rinascimento, Florence, SISMEL/ Edizioni del Galluzzo, 2008, p. 35-83.

19 Au lieu d'expliquer le succès du français par son prestige culturel, Simon GaunT (Marco Polo's "Le devisement du monde". Narrative Voice, Language and Diversity, Cambridge (R.-U.), D. S. Brewer, 2013) souligne que le français est utilisé comme langue véhiculaire pour des raisons pragmatiques dans des réseaux mercantiles.

20 Dante Alighieri, De l'éloquence en vulgaire, éd. cit., I, X, 2, p. 120-121.

21 Max Pfister («Le bilinguisme de Brunetto Latini: le Liore du Trésor», dans Claire 
Kappler, Suzanne Thiolier-MÉJeAn (dir.), Le Plurilinguisme au Moyen Âge. Orient-Occident: de Babel à la langue une, L'Harmattan, 2009, p. 203-216) montre comment se comporte Brunetto Latini à l'égard d'une source latine (Cicéron) qu'il traduit en langue d'oïl et en italien: les latinismes filtrent davantage dans la langue italienne, plus servilement dépendante de son modèle, tandis que se formulent des expressions plus émancipées du latin dans l'usage $d u$ français sur fond d'une production de binômes synonymiques (dittologie).

22 Serge VAnvolsem, art. cit., p. 31 ; Paolo Divizia, "Aggiunte (e una sottrazione) al censimento dei codici delle versioni italiane del "Tresor" di Brunetto Latini ", Medioevo Romanzo, 2008, p. 377-394. Sur le volgarizzamento du Tresor réalisé par un prisonnier pisan à Gênes (Florence, Biblioteca Medicea Laurenziana, Pluteus, XLII.23), voir entre autres David NAPOLITANo, «Bondì Testario's bewerking van Brunetto Latini's Li Livres dou Tresor ", Incontri. Rivista europea di studi italiani, vol. 26, $\mathrm{n}^{\circ}$ 1, 2011, p. 3-15, DOI : 10.18352 /incontri.814.

23 Voir Andrea ZorZI, «I letterati ambasciatori nel tardo Medioevo ", dans Sergio Luzzatto, Gabriele Pedullà (dir.), Atlante della letteratura italiana, vol. I, Amedeo DE VINCENTIIs (dir.), Dalle origini al Rinascimento, Turin, G. Einaudi, 2010, p. 282-285.

24 Tesoretto, livre II, v. 32.

25 Tresor, éd. cit., II, 84, p. 528.

26 Par exemple Alison CoRnIsH (op. cit.) suggère sans réelle preuve solide que Brunetto Latini, de retour d'exil, aurait importé en Italie les Faits des Romains.

27 Voir Isabelle Heullant-Donat, Marie-Anne Polo de BeAulieu, "Histoire d'une traduction: Le Livre de l'Échelle de Mahomet", dans Le Livre de l'Échelle de Mahomet, trad. de Gisèle Besson et Michèle Brossard-Dandré, Librairie générale française (Le Livre de poche), 1991, p. 11-26 ; María Rosa Menocal, The Arabic Role in Medieval Literary History. A Forgotten Heritage, Philadelphie, University of Pennsylvania Press, 1987, p. 122-124.

28Chris Wiскнам, Sleepwalking into a New World. The Emergence of Italian City Communes in the Twelfth Century, Princeton University Press, 2015. 